\title{
Immersive Virtual Reality as a Rehabilitative Technology for Phantom Limb Experience: A Protocol
}

\author{
CRAIG D. MURRAY, ${ }^{1}$ EMMA PATCHICK, ${ }^{1}$ STEPHEN PETTIFER, ${ }^{2}$ \\ FABRICE CAILLETTE, ${ }^{2}$ and TOBY HOWARD ${ }^{2}$
}

\begin{abstract}
This paper describes a study protocol to investigate the use of immersive virtual reality as a treatment for amputees' phantom limb pain. This work builds upon prior research using mirror box therapy to induce vivid sensations of movement originating from the muscles and joints of amputees' phantom limbs. The present project transposes movements of amputees' anatomical limbs into movements of a virtual limb presented in the phenomenal space of their phantom limb. It is anticipated that the protocol described here will help reduce phantom limb pain.
\end{abstract}

\section{INTRODUCTION}

$\mathbf{F}^{\mathrm{c}}$ OLLOWING AMPUTATION, the patient commonly experiences their lost limb as still intact. ${ }^{1}$ These phantom limbs can often be painful, ${ }^{2}$ which is a large and pervasive problem in many amputees' lives. Adjustment to amputation is negatively correlated with phantom limb pain (PLP), ${ }^{3}$ with affected amputees less likely to use a prosthetic limb ${ }^{4}$ resulting in the restriction of normal activities and higher levels of depression. ${ }^{5}$

One promising development in the treatment of PLP is the mirror box: a device created by placing a mirror inside a box in such a way as to allow amputees to view a reflection of their anatomical limb in the visual space occupied by their phantom limb. ${ }^{6}$ For some amputees the box is able to induce vivid sensations of movement originating from the muscles and joints of their phantom limb, with some patients having their PLP relieved and others able to gain control over paralyzed phantoms. ${ }^{6,7}$

There are, however, limitations imposed when using the mirror box. The patient is required to focus on the reflection of their intact arm in order to receive illusory visual feedback of their phantom limb. However, it only takes a look at the intact arm providing the reflection to break this visual illusion. Patients must also remain in a fixed position, with their head oriented towards the mirror and their torso in mid-saggital plane with the mirror so as not to alter the reflection of their limb. The work on the mirror box is, however, greatly promising and suggests that other visual therapies that work in similar ways, whilst overcoming these drawbacks, may improve upon the therapeutic benefits observed.

The present research uses immersive virtual reality (IVR) to transpose the movements made by an amputee's remaining anatomical limb into movements of a virtual limb in the phenomenal space occupied by their phantom limb. This provides a similar illusion to the mirror box without the confines imposed by reflection-based work: in the virtual environment only the virtual phantom limb moves so the illusion is robust, independent of the orientation or focus of the patient. Considering the relatively nascent approach of this system, and the nature of pain treatment in general, a control group is necessary to assess the outcome of treatment over and above any placebo effects. There-
Au: Please provide academic degree (e.g., M.D., Ph.D.) for each author.

${ }^{1}$ School of Psychological Sciences and ${ }^{2}$ School of Computer Science, University of Manchester, Manchester, United Kingdom. 
fore, this transposition of movement does not take place for a control group: movements of the anatomical limb only generate movements in the virtual, corresponding limb.

The objectives of the work are to (1) produce virtual facsimiles of amputees phantom limbs that can be controlled by movements of the opposite anatomical limb; and (2) assess the efficacy of IVR in the treatment of PLP, in decreasing body image dissatisfaction, and in enabling successful prosthesis use.

\section{PROTOCOL}

\section{Study design}

The study is a longitudinal one and has a between-subjects design. There is one independent variable, as described above-namely whether participants use a virtual representation of their phantom limb controlled by movements made by their opposite anatomical limb (group A) or a virtual representation of their intact limb controlled by movements made by the corresponding anatomical limb (group B). It is hypothesized that group A will experience significant short- and long-term positive changes in the frequency and severity of PLP, psychosocial issues, activity restriction, and satisfaction with a prosthesis, while group B will not.

\section{Participants}

Participants are recruited through a sub-regional Disablement Services Centre in the United Kingdom. The inclusion criteria are being a unilateral adult amputee, having phantom limb pain, and a minimum of 12 months post-amputation. Partici- pants vary along such dimensions as age, sex, whether their missing limb is upper or lower, and the type of prosthetic used. No participants with serious visual or cognitive impairments are recruited. A target sample of 32 amputees are randomly assigned to the experimental $(n=16)$ and control groups $(n=16)$. Both groups have a standardized visual representation of their body, including the phantom limb.

\section{Materials}

A V6 VR head-mounted display (HMD) is used to present the virtual environment (VE). In order to represent participants' limb movements a 5DT-14 data glove and sensors are used for upper-limb amputees, while sensors are used for lower-limb amputees. Sensors are attached to the elbow and wrist joints or the knee and ankle joints for upper- and lower-limb amputees respectively. A Polhemus Fastrak monitors head and limb movements.

The minimal VE represents the participant from an embodied point-of-view (Fig. 1). Participants are provided with a number of tasks (described below) in this VE in order to provide opportunities for hand-eye or foot-eye coordination of their virtual limb.

The sensors are used to control a model of the human body. Placing constraints on the joint angles allows impossible poses to be avoided and transferring a movement from one limb to another is possible due to the joint angles parameterization. For example, once the joint angles are recovered from the right arm through inverse kinematics, applying these joints angles to the left arm results in mirroring the movement. This method of transferral as well as other implemented software generates responsive, fluent, real-time motion, allowing

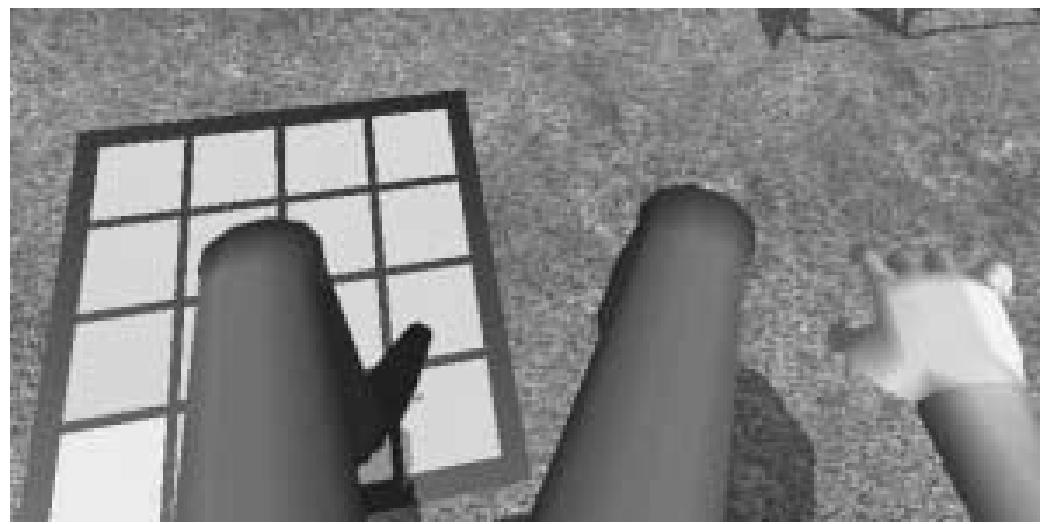

FIG. 1. One possible view participants may see when taking part in the experiment. 
virtual limbs to move in synchrony with anatomical limbs.

The appearance of the body is modeled by a deformable polygonal mesh, attached onto the underlying kinematic model. Whilst the mesh-skinning gives realistic results at a gross level, there are certain constraints imposed on the level of detail at which the virtual limb can be presented. Features such as fingernails and muscle tone are omitted from the virtual body (Fig. 1). However, the interface on start-up does allow the color of skin and clothes to be altered to approximate those of the participant.

\section{Experimental measures}

Each of the following measures are completed by participants a total of two times: 1 week prior to using the VE and once on completion of involvement with the study:

1. The McGill Pain Questionnaire $(\mathrm{MPQ})^{8}$ is administered in order to indicate participants' subjective phantom pain experience.

2. The Amputee Body Image Scale (ABIS) ${ }^{9}$ assesses levels of body image disturbance.

3. The Trinity Amputation and Prosthesis Experience Scales (TAPES) ${ }^{10}$ is a self-report instrument designed to measure practical and psychosocial adjustment to an artificial limb.

A short-form of the MPQ is administered at the end of each IVR session in order to give a continuous assessment of pain levels and pain diaries are completed by participants throughout the course of the study to allow a more contextualised analysis of participant's phantom pain experience. A vividness of imagery scale is used to measure participants' ability to visualise movement in their phantom limb during IVR sessions.

It is also important to build a more qualitative understanding of participant's experience of using the IVR system and of their phantom limb experience in their own words, given that it is often highly unique and subjective. To enable this, participants take part in semi-structured interviews throughout the course of their involvement in the research. It is envisaged that by combining data from qualitative and quantitative measures, exploratory analysis will inform the best protocol for future research.

\section{Procedure}

Over a twelve-week period, each participant uses the IVR equipment every two weeks for a pe- riod of 30 minutes. Participants complete four tasks (in repetitions): placing their virtual phantom hand or foot on tiles which light up in a random sequence; batting or kicking a virtual ball; tracking the motion of a moving stimulus which requires raising and bending the limb; and directing a virtual ball towards a target. Group B complete the same tasks as group A, but with the movements of their physical limbs being transposed onto the movements of their corresponding virtual limb rather than their virtual phantom limb.

\section{Data collection and analysis}

Participants' scores obtained on the MPQ, ABIS, and TAPES are compared over the study period and between the two IVR conditions. This data analysis allows judgements to be made regarding the short and long-term therapeutic benefit of IVR for phantom pain relief, body image disturbance and prosthesis satisfaction.

\section{CONCLUSION}

It is anticipated that our protocol will help reduce phantom limb pain.

\section{ACKNOWLEDGMENTS}

The work reported in this paper is supported by a grant from Remedi Rehabilitation.

\section{REFERENCES}

1. Murray, C.D. (2004). An interpretative phenomenological analysis of the embodiment of artificial limbs. Disability and Rehabilitation 26:963-973.

2. Ramachandran, V.S., \& Hirstein, W. (1998). The perception of phantom limbs, Brain 121:1603-1630.

3. Katz, J. (1992). Psychophysiological contribution to phantom limbs. Canadian Journal of Psychiatry 37:282298.

4. Dolezal, J.M., Vernick, S.H., Khan, N., et al. (1998). Factors associated with use and nonuse of an AK prosthesis in a rural, southern, geriatric population. International Journal of Rehabilitation and Health 4:245251.

5. Williamson, G.M., Schulz, R., Bridges, M.W., et al. (1994). Social and psychological factors in adjustment to limb amputation. Journal of Social Behavior and Personality 9:249-268.

6. Ramachandran, V.S., \& Rogers-Ramachandran, D. (1996). Synaesthesia in phantom limbs induced with 
mirrors. Proceedings of the Royal Society of London-B Biological Sciences 263:377-386.

7. Brodie, E.E., Whyte, A., \& Waller, B. (2003). Increased motor control of a phantom leg in humans results from the visual feedback of a virtual leg. Neuroscience Letters 341:167-169.

8. Melzack, R. (1975). The McGill pain questionnaire: major properties and scoring methods. Pain 1:277-279.

9. Breakey, J.W. (1997). Body image: The lower-limb amputee. Journal of Prosthetics and Orthotics 9:58-66.

10. Gallagher, P., \& MacLachlan, M. (2000). Development and psychometric evaluation of the Trinity
Amputation and Prosthesis Scales (TAPES). Rehabilitation Psychology 45:130-154.

Address reprint requests to:

Dr. Craig D. Murray

School of Psychological Sciences Au: Please

University of Manchester provide Manchester, UK complete

mailing ad-

E-mail: craig.murray-2@manchester.ac.uk dresses for reprints. 